\title{
A sleep-regulatory circuit integrating circadian, homeostatic and environmental information in Drosophila
}

\author{
Angélique Lamaze ${ }^{1}$, Patrick Krätschmer ${ }^{1}$ and James E. C. Jepson ${ }^{1,2 *}$ \\ ${ }^{1}$ Department of Experimental and Clinical Epilepsy, UCL Institute of \\ Neurology, UK \\ ${ }^{2}$ Lead Contact \\ *Correspondence: j.jepson@ucl.ac.uk
}

\section{SUMMARY}

In the wild, when to go to sleep is a critical decision. Sleep onset is controlled by two processes: the circadian clock, and a homeostat measuring sleep drive $[1,2]$. Environmental stimuli must also clearly intersect with the circadian clock and/or homeostat so that sleep is initiated only when appropriate. Yet how circadian, homeostatic and environmental cues are integrated at the circuit level is unclear. Recently, we found that DN1p clock neurons in Drosophila act to prolong morning wakefulness at elevated ambient temperatures [3]. Here we show that a subset of DN1p neurons exhibit temperature-sensitive increases in excitability, and define an output pathway linking DN1p neurons to downstream sleep-regulatory circuits. We show that DN1p neurons project axons to a subdomain of the Anterior Optic Tubercle (AOTU), and here make inhibitory synaptic connections with sleep-promoting tubercular-bulbar (TuBu) neurons. Using unbiased trans-synaptic labeling, we show that these TuBu neurons form synaptic connections with R-neurons innervating the ellipsoid body, subsets of which control homeostatic sleep drive [4]. DN1p excitability is clock-dependent, peaking in the late night and 
early morning [5]. Thus, integration of circadian and thermo-sensory

information by DN1p neurons and subsequent inhibition of sleep-promoting

TuBu neurons provides a mechanism by which an environmental stimulus can

regulate sleep onset during a specific compartment of the day-night cycle.

Furthermore, our results suggest that the AOTU functionally links circadian

and sleep homeostat circuits in Drosophila. 


\section{RESULTS AND DISCUSSION}

\section{Consolidated sleep onset in Drosophila is gated by circadian and}

\section{thermal cues}

Using the standard definition of a Drosophila sleep bout as a $5 \mathrm{~min}$ period of inactivity (measured using the Drosophila Activity Monitor (DAM) system [6]), we recently showed that elevating ambient temperature from $22^{\circ} \mathrm{C}$ to $\geq 30^{\circ} \mathrm{C}$ delays onset of the first morning sleep bout in Drosophila males [3]. However, Drosophila sleep is often initially fragmented during the early morning at lowto-medium temperatures (Figure $1 \mathrm{~A}$ ). To gain a more robust view of how temperature changes affect consolidated sleep, we generated an R-based program capable of quantifying up to 30 distinct sleep parameters using raw DAM system data (see STAR Methods). We used this program to analyse how various properties of sleep architecture were modulated by temperature increases (Figure S1A-J), particularly the daytime longest sleep bout (dLSB).

Given the prolonged inactivity during the dLSB (Figure 1A), we reasoned that this parameter would yield a more accurate representation of when consolidated sleep was occurring. We measured the onset and offset of the dLSB in control flies and in mutants with short and long circadian periods $\left(p e r^{S}\right.$ and per ${ }^{\perp}$ [7]. We observed an advance in the onset of the dLSB in pers mutants and a delay in per relative to control flies at $22^{\circ} \mathrm{C}$ (Figure $1 \mathrm{~B}$ ) while offset of the dLSB was similarly advanced in per $r^{S}$ mutants (Figure S1A), demonstrating that timing of the dLSB is clock-dependent. The timing of the dLSB was also temperature-sensitive. Shifting flies from $22^{\circ} \mathrm{C}$ to $\geq 30^{\circ} \mathrm{C}$ resulted in a significant delay in both the onset and offset of the dLSB (Figures 1A, C and Figure S1B). Importantly, delayed onset at $\geq 30^{\circ} \mathrm{C}$ was 
suppressed in male flies lacking a functional circadian clock $\left(\mathrm{tim}^{\mathrm{KO}}\right)$ (Figure 1D) [3]. Thus, circadian and thermo-sensory pathways interact to modulate onset of the dLSB.

\section{Thermo-sensitive DN1p neurons delay morning sleep}

Which clock neurons influence delay of the dLSB at high temperature? DN1p clock neurons promote delay of the first morning sleep bout at high temperatures [3]. Thus, we tested whether synaptic output from DN1p neurons was also required to delay the $\mathrm{dLSB}$ at $31^{\circ} \mathrm{C}$. To suppress synaptic output from DN1p neurons, we expressed a temperature-sensitive inhibitor of endocytosis (shi[ts]) using the R18H11-Gal4 driver, which labels 7-8 DN1p neurons $[8,9]$. Expression of shi[ts] blocks synaptic release at $31^{\circ} \mathrm{C}$ but not $22^{\circ} \mathrm{C}$ via inhibition of synaptic vesicle recycling, facilitating acute inhibition of DN1p output at high temperature. As expected, control flies exhibited a delay in the onset of the $\mathrm{dLSB}$ at $31^{\circ} \mathrm{C}$ compared to $22^{\circ} \mathrm{C}$ (Figure 1E, G). In contrast, inhibiting synaptic release from $R 18 H 11$-positive DN1p neurons blocked this effect (Figure 1F, G). Both the delay in onset and reduction in length of the longest night sleep bout at $31^{\circ} \mathrm{C}$ relative to $22^{\circ} \mathrm{C}$ was unaffected by DN1p inhibition (Figure 1E, F and Figure S1K-M). DN1p neurons therefore modulate the timing of consolidated sleep during the day but not the night in response to temperature changes.

We next asked whether the excitability of DN1p neurons was temperature-sensitive. To examine this, we expressed CAMPARI in DN1p neurons. When stimulated with UV light, the CAMPARI fluorophore undergoes green-to-red photo-conversion in high intracellular calcium, yielding an optical 
read-out of neuronal activity [10]. We utilised the relatively calcium-insensitive V398D variant to increase the dynamic range of CAMPARI [10], and performed photo-conversion experiments at Zeitgeber Time (ZTO-2) either at $22^{\circ} \mathrm{C}$ or following a shift from $22^{\circ} \mathrm{C}$ to $31^{\circ} \mathrm{C}$ at ZTO. Following photoconversion, we measured the ratio of non- and photo-converted CAMPARI fluorescence in DN1p cell bodies (Figure 1H-J). At ZT0-2 and $22^{\circ} \mathrm{C}$, approximately half of $R 18 H 11-\mathrm{DN} 1 \mathrm{p}$ neurons exhibited robust levels of photoconverted CAMPARI, indicative of high neuronal excitability (Figure $1 \mathrm{H}, \mathrm{J})$. In contrast, at the same time-point but at $31^{\circ} \mathrm{C}$, we detected a more uniform increase in CAMPARI photo-conversion (Figure 1I, J). Indeed, the coefficient of variation of the ratio of photo-converted to non-converted CAMPARI was higher at $22^{\circ} \mathrm{C}$ relative to $31^{\circ} \mathrm{C}(0.65$ vs. 0.32$)$, and the population variance at $22^{\circ} \mathrm{C}$ was also significantly higher relative to $31^{\circ} \mathrm{C}(p=0.026$, F-test $)$. These results suggest that DN1p clock cells contain a thermo-sensitive subpopulation that act in a wake-promoting circuit in the morning.

\section{DN1p neurons form synaptic connections in the Anterior Optic Tubercle}

To identify relevant circuits downstream of DN1p neurons, we co-expressed distinct fluorophores localised to presynaptic and dendritic domains (UAS-sytGFP and UAS-DenMark respectively) using R18H11-Gal4 (Figure 2A).

Confocal imaging revealed DN1p dendrites in the dorsal posterior protocerebrum, within the region in which thermo-sensory TrpA1-expressing neurons form synaptic contacts with DN1p neurons [3]. DN1p presynaptic boutons innervated two neuropil regions: the pars intercerebralis [11], but also the Anterior Optic Tubercle (AOTU) (Figure 2A). Expression of syt-GFP using 
a distinct driver that also labels DN1p neurons (clk4.1M-Gal4) confirmed the presence of DN1p synapses in this region (Figure S2A) $[12,13]$.

The AOTU is a component of the Anterior Visual Pathway, linking neurons in the optic lobe medulla to the Ellipsoid Body (EB) [14], a domain of the central complex involved in motor control [15], navigational learning and orientation [16, 17], and importantly, homeostatic sleep drive [4]. Dendrites from tubercular-bulbar (TuBu) neurons innervate the AOTU and project axons to the bulb (also known as the lateral triangle), where they synapse onto EB ring (R-) neurons $[14,18]$. The AOTU can be divided into distinct regions: the medial (AOTUm), intermediate medial (AOTUim), intermediate lateral (AOTUil) and lateral (AOTUla) (as defined in ref. [14]) (Figure 2A). DN1p synapses predominantly localise to the AOTUil, with a small proportion innervating the AOTUim and AOTUla (Figure 2A). We identified TuBu neurons innervating the AOTUil that can be labelled using the R92H07-Gal4 driver (Figure 2B). To confirm synaptic connectivity between DN1p and R92H07-TuBu neurons, we first used orthogonal LexA- and Gal4-drivers to express different fluorophores in each cell type, revealing close association of DN1p axons/synapses with R92H07-TuBu dendrites (Figure 2B). Using the same approach we next performed GRASP experiments by expressing complementary fragments of GFP in DN1p and R92H07-TuBu neurons [19]. Confocal imaging revealed reconstituted GFP fluorescence specifically in the AOTUil region (Figure 2C and Figure S2B), suggesting synaptic connectivity between DN1p and TuBu neurons.

We also tested whether DN1p neurons formed connections with TuBu neurons in the more lateral region of the AOTU (AOTUla). Using the R83H09- 
Gal4 driver we labelled AOTUla TuBu neurons (Figure S2E). DN1p synapses/axons tiled the boundary of the dendritic domain formed by R83H09-Tubu neurons (Figure S2C). However, in contrast to R92H07-Tubu neurons in the AOTUil, no GRASP signal between DN1p and R83H09-Tubu neurons in the AOTUla was observed (Figure S2D). Thus, DN1p neurons appear to physically associate predominantly with TuBu neurons in the AOTUil region.

\section{DN1p neurons inhibit TuBu neurons}

We next examined whether DN1p and R92H07-TuBu neurons are functionally connected. The excitability of DN1p neurons oscillates in a clock-dependent manner, with a peak in the late night/early morning and a trough in the late day/early night [5]. Thus, we wondered whether the excitability of R92H07TuBu neurons also oscillated, and if so, whether such oscillation was positively or negatively correlated with DN1p neurons. To do so, we again utilised CAMPARI [10]. Consistent with previous patch-clamp analysis [5], excitability of DN1p neurons at $31^{\circ} \mathrm{C}$ was higher in the morning (a period of increased wakefulness) relative to the afternoon when male flies are largely asleep (Figure 3A). In R92H07-TuBu neurons the opposite was the case (Figure 3B). This suggests that DN1p neurons inhibit R92H07-TuBu neurons. To test this, we expressed ChannelRhodopsin2-XXL (ChR2-XXL) in DN1p neurons and the GCamP6s reporter of intracellular calcium in $R 92 H 07-T u B u$ neurons [20, 21]. We optogenetically activated DN1p neurons at ZT9 and $31^{\circ} \mathrm{C}-$ a time-point and temperature at which excitability of DN1p neurons is low and R92H07-TuBu neurons high (Figure 3A, B) [5] - and measured 
changes in intracellular calcium in R92H07-TuBu neurons. In control brains expressing GCamP6s in R92H07-TuBu neurons, excitation with UV light causes an increase in GCamP6s fluorescence due to an overlap with the GCamP6s excitation spectra (Figure 3C). However, when UV light-activated ChR-XXL was simultaneously expressed in DN1p neurons, this increase in GCamP6s fluorescence was greatly reduced, indicative of a parallel reduction in intracellular calcium (Figure 3C). Collectively, the above results indicate that DN1p neurons inhibit R92H07-TuBu neurons.

\section{TuBu neurons in the AOTUil are sleep-promoting}

DN1p neurons are wake-promoting during the morning at high temperatures [3] (Figure 1F). Thus, if DN1p neurons inhibit R92H07-TuBu neurons, R92H07-TuBu neurons should promote sleep. To test this, we expressed TrpA1 (a temperature-gated cation channel) in R92H07-neurons and measured sleep following a shift from $22^{\circ} \mathrm{C}$ (a non-activating temperature) to $31^{\circ} \mathrm{C}$ (an activating temperature) [22]. Excitation of R92H07-neurons immediately and profoundly induced sleep throughout the day, whereas control flies exhibit prolonged morning wakefulness (Figure 3D, E). Sleep loss during the night caused by elevated temperature was also suppressed by R92H07-TuBu neurons activation (Figure 3D). Note that at the end of the night, morning anticipation (a clock-driven locomotor behavior) remained intact following R92H07-TuBu excitation (Figure 3D, grey arrow). In addition, the total number of sleep bouts during the night was not significantly reduced (Figure S3A, B). Thus, these flies are able to exhibit waking behavior and are not simply paralyzed. On the following recovery day at $22^{\circ} \mathrm{C}$, flies in which 
sleep was previously initiated via R92H07-TuBu excitation exhibited a strong 'negative rebound', showing a reduction in the length of the dLSB relative to the initial control day at $22^{\circ} \mathrm{C}$, in contrast to an increase in controls (Figure 3D, F). These results suggest that $R 92 H 07-T u B u$ neurons are coupled to circuits that control homeostatic sleep drive, in contrast to other sleep-modulatory celltypes in the fly brain such as octopaminergic neurons [23]. To test whether suppressing R92H07-TuBu output impacted sleep at high temperature, we acutely blocked synaptic release from R92H07-TuBu neurons using shi[ts] (Figure S3C). Inhibition of R92H07-TuBu neurons reduced the length of the longest sleep bout during the day but not the night (Figure S3D-I), consistent with a role for $\mathrm{R} 92 \mathrm{H} 07-\mathrm{TuBu}$ neurons in promoting day sleep. We note, however, that the effect of inhibiting R92H07-TuBu neurons on overall sleep architecture was subtle compared to acute activation (Figure S3E, Figure 3D). These results support a model in which additional sleep-promoting TuBu neurons within the AOTUil are unmarked by the R92H07-Gal4 driver and are still available to promote sleep during inhibition of $R 92 H 07-T u B u$ neurons.

\section{Sleep-regulatory TuBu neurons form synaptic connections with diverse}

\section{ellipsoid body R-neurons}

Finally, we sought to identify circuits downstream of R92H07-TuBu neurons.

To do so, we utilised a recently devised system for unbiased trans-synaptic labelling: trans-Tango [24]. This method involves expression of a signalling molecule (Glucagon) tethered to presynaptic domains of a neuron of interest. In parallel, a post-synaptic Glucagon receptor is expressed in all neurons, and is connected to a signalling pathway yielding transcription of the fluorophore 
mtdTomato following binding of Glucagon to its receptor on post-synaptic neurons (see ref. 24 for details). Presynaptic neurons are simultaneously labelled by expressing a distinct fluorophore (myr-GFP) [24]. We applied this method to R92H07-TuBu neurons. Control flies containing trans-Tango but no driver exhibited non-specific mtdTomato fluorescence in the antennal mechanosensory and motor centre (Figure 3G). In contrast, when trans-Tango was driven by R92H07-Gal4, we observed overlapping myr-GFP and mtdTomato fluorescence in the superior region of the bulb (Figure $3 \mathrm{H}, \mathrm{I}$ ), as indicated by the dorsal location of myr-GFP and mtdTomato relative to the EB midline (Figure 3I). Neurons post-synaptic to R92H07-TuBu neurons projected to the EB (Figure 3I, J). From the location of their dendrites in the superior bulb and axonal projections in the outer segments of the EB, these may represent R2-, R4d- and R5 neurons (Figure 3I) [14, 17, 18]. R1/R3 neurons in the central domain of the EB were also visible, albeit more weakly (Figure 3I). However, since the dendrites of R1/R3 neurons innervate the inferior bulb [14, 18], these are unlikely to be functionally connected to $R 92 \mathrm{H} 07-\mathrm{TuBu}$ neurons, which project to the superior bulb (Figure 3I). In summary, our results define a tripartite sleep-regulatory circuit linking DN1p neurons to the EB via the AOTU.

\section{Conclusions}

Here we elucidate a circuit mechanism by which sleep during the early morning is suppressed at elevated temperatures. We propose that circadian and thermo-sensory information is concurrently encoded in the excitability of DN1p clock neurons. Subsequently, sleep-regulatory information is transmitted from DN1p neurons to the EB, a motor control centre $[15,25]$, via TuBu 
neurons innervating the AOTUil. This results in the suppression of locomotion resulting from sensory input such as visual stimuli and initiation of sleep $[4,17$, 26]. Our trans-synaptic labelling experiments linking TuBu to EB R-neurons are consistent with recent studies defining these two cell-types as neighboring modules of the anterior visual pathway $[14,18]$. Importantly, R2-neurons also receive recurrent feedback from sleep-promoting neurons in the dorsal fanshaped body [26], and enhance sleep drive when artificially activated [4]. R2neurons have therefore been proposed to act as a component of the sleep homeostat [4]. R92H07-TuBu neurons form synaptic contacts with R-neurons innervating multiple EB rings (Figure 3I). Thus, the sleep-promoting action of R92H07-TuBu neurons is likely to occur via simultaneous modulation of multiple subsets of EB neurons, the net effect of which is to suppress locomotion and promote sleep. The inhibition of $R 92 H 07-T u B u$ neurons by DN1p neurons provides a mechanism to promote wakefulness when DN1p activity is high: during the early morning and at high temperatures (Figures 1 I and $3 A$ ) $[3,5]$. In concert with previous data $[3,4]$, our results suggest that the DN1p > TuBu > R-neuron circuit represents a physical link between clock, sensory, and sleep homeostat neurons, enabling circadian and environmental cues to dynamically regulate sleep onset. 


\section{FIGURE LEGENDS}

\section{Figure 1: Thermo-sensitive DN1p neurons are required for delaying} sleep onset at elevated temperature.

(A) Upper panel: protocol used for temperature-shift experiments. Flies were housed under $12 \mathrm{~h}$ light: $12 \mathrm{~h}$ dark (12L: 12D) conditions. Sleep was measured on two consecutive days at ambient temperatures of $22^{\circ} \mathrm{C}$ and $31^{\circ} \mathrm{C}$ respectively. Light bars: day. Dark bars: night. Lower panels: sleep bouts for a single male fly under the above conditions. Note the fragmented sleep early in the morning at $22^{\circ} \mathrm{C}$, before a longer consolidated sleep bout (grey bar). dLSB: longest sleep bout. At $31^{\circ} \mathrm{C}$, morning wakefulness is enhanced, and onset of the dLSB is delayed.

(B) Onset of the dLSB in iso control males, per $r^{S}$ and per mutants at $22^{\circ} \mathrm{C}$. In these and all subsequent Tukey box plots, boxes show the $25^{\text {th }}$, median and $75^{\text {th }}$ percentiles. Whiskers show $1.5 x$ the interquartile range. Dots represent outliers. pers: $\mathrm{n}=30$; iso: $\mathrm{n}=31$; per $\mathrm{L} \mathrm{n}=37$.

(C) Onset of the dLSB in iso controls at different ambient temperatures. $22^{\circ} \mathrm{C}$ : $n=82 ; 30^{\circ} \mathrm{C}: \mathrm{n}=35 ; 31^{\circ} \mathrm{C}: \mathrm{n}=47$

(D) Onset of the dLSB in timeless knockout $\left(\operatorname{tim}^{\mathrm{KO}}\right)$ mutants. $22^{\circ} \mathrm{C}: \mathrm{n}=85$; $30^{\circ} \mathrm{C}: \mathrm{n}=33 ; 31^{\circ} \mathrm{C}: \mathrm{n}=52$

(E) Overlaid mean sleep levels across two consecutive $24 \mathrm{~h}$ periods at $22^{\circ} \mathrm{C}$ and $31^{\circ} \mathrm{C}$ in control adult male flies harbouring the UAS-shi[ts] transgene alone $(+>$ shi[ts]). Note the delay in the onset of sleep during the day at elevated temperature (arrow). Error bars represent SEM.

(F) Mean sleep levels in adult male flies following acute inhibition of DN1p synaptic output $(\mathrm{R} 18 \mathrm{H} 11>$ shi[ts] $)$ at $31^{\circ} \mathrm{C}$ compared to the previous day at 
$22^{\circ} \mathrm{C}$ where DN1p synaptic output was uninhibited. Note the absence of a delay in morning sleep onset at $31^{\circ} \mathrm{C}$ (arrow).

(G) Box plots showing median difference in onset of the dLSB following shifts from $22^{\circ} \mathrm{C}$ to $31^{\circ} \mathrm{C}$ in control and experimental male flies. $R 18 H 11>+: \mathrm{n}=63$; $+>$ shi[ts]: $\mathrm{n}=50 ; R 18 H 11>$ shi[ts]: $\mathrm{n}=63$.

$(\mathrm{H}, \mathrm{I})$ Confocal images showing non-converted and photo-converted CAMPARI in R18H11-positive DN1p neurons at ZT0-2 at either $22^{\circ} \mathrm{C}(\mathrm{H})$ or $31^{\circ} \mathrm{C}(\mathrm{I})$. Arrows point to $\mathrm{R} 18 \mathrm{H} 11$-positive DN1p neurons where robust photoconversion, and thus elevated neuronal excitability, can be detected. Scale bars, $50 \mu \mathrm{m}$.

(J) Dot plot illustrating ratios of non-converted to photo-converted CAMPARI in $\mathrm{R} 18 \mathrm{H} 11$-positive DN1p neurons at either $22^{\circ} \mathrm{C}$ or $31^{\circ} \mathrm{C}$. At $22^{\circ} \mathrm{C}$, two subpopulations are apparent (minimal or robust photo-conversion), whereas at $31^{\circ} \mathrm{C}$ DN1p neurons show a higher and more uniform degree of CAMPARI photo-conversion. $22^{\circ} \mathrm{C}: \mathrm{n}=26$ neurons from 4 brains. $31^{\circ} \mathrm{C}: \mathrm{n}=37$ neurons from 7 brains.

${ }^{*} p<0.05,{ }^{* *} p<0.01,{ }^{* * *} p<0.001$, ns - not significant, Kruskal Wallis test with Dun's post-hoc test. See also Figure S1. 
Figure 2. DN1p neurons form synaptic contacts with TuBu neurons in the AOTU.

(A) Upper panel: confocal images showing co-expression of presynaptic and dendritic markers in adult male DN1p neurons. Arrows: pre-synaptic SYTGFP puncta in the anterior optic tubercle (AOTU). Lower panel: highresolution image of DN1p presynaptic domains (arrow) in the AOTU. Subdomains of the AOTU are as follows. La: lateral, il: intermediate lateral, im: intermediate medial, m: medial [14]. BRP: Bruchpilot, a presynaptic neuropil marker.

(B) Upper panel. Confocal images showing projection patterns of DN1p neurons (labelled using R18H11-LexA) and R92H07-Gal4-positive TuBu neurons. Distinct membrane-tagged fluorophores were expressed in each cell type. Arrows point to the AOTU region. Lower panel: high-resolution image of the AOTU showing overlap (arrows) between DN1p axons/synapses and dendritic regions of $\mathrm{R} 92 \mathrm{H} 07-\mathrm{TuBu}$ neurons.

(C) High-resolution confocal image showing synaptic connectivity between DN1p and R92H07- TuBu neurons, as demonstrated through GRASP. Arrows point to reconstituted GFP signal formed in the AOTUil following expression of membrane-tagged GFP fragments in DN1p and R92H07-TuBu neurons.

Scale bars: $50 \mu \mathrm{m}$. See also Figure S2. 


\section{Figure 3. DN1p neurons inhibit sleep-promoting TuBu neurons.}

(A-B) Box plots showing ratios of photo-converted (red) and non-converted (green) CAMPARI fluorescence at the stated time-points in R18H11-DN1p (A) and R92H07-TuBu neurons (B). R18H11-DN1p neurons (A): ZT0-1, n = 37 neurons from 7 brains; ZT7-8, $n=52$ neurons from 8 brains. R92H07-TuBu neurons (B): ZT0-1, $\mathrm{n}=51$ neurons from 7 brains; ZT6-7, $\mathrm{n}=75$ neurons from 8 brains.

(C) Left panel: mean change in GCamP6s fluorescence in R92H07-TuBu neurons (R92H07-LexA > LexAop-GCamP6s) following UV light activation of ChR2-XXL in DN1p neurons (grey; R18H11-Gal4 > UAS-ChR2-XXL), or solely due to UV light stimulation (black). Error bars represent SEM. Right panel: dot plots of individual cellular changes in GCamP6s fluorescence following UV light stimulation in the presence (grey) or absence (black) of ChR2-XXL expressed in DN1p neurons. Mean and SEM are shown.

(D) Overlaid mean sleep levels across three consecutive $24 \mathrm{~h}$ periods at $22^{\circ} \mathrm{C}$ (control day), $31^{\circ} \mathrm{C}\left(\operatorname{TrpA} 1\right.$ activation) and $22^{\circ} \mathrm{C}$ (recovery day), in the stated genotypes. Arrow: reduced sleep prior to lights-on during R92H07-TuBu activation. Error bars represent SEM.

$(E, F)$ Box plots showing median difference in the onset of the dLSB following shifts from $22^{\circ} \mathrm{C}$ to $31^{\circ} \mathrm{C}(\mathrm{E})$ or in the length of the dLSB between control and recovery days $(F)$ in control and experimental male flies. $R 92 H 07>+: n=29$; + > TrpA1: $\mathrm{n}=34 ; R 92 H 07>\operatorname{TrpA1}: \mathrm{n}=36$.

${ }^{* *} p<0.01,{ }^{* * *} p<0.001$, Mann-Whitney U-test $(A-C)$ or Kruskal Wallis test with Dun's post-hoc test (E, F). 
(G) Confocal image of a control trans-Tango male adult brain lacking any Gal4 driver. BRP, Bruchpilot.

$(\mathrm{H}, \mathrm{I})$ Confocal images of trans-Tango driven by $\mathrm{R} 92 \mathrm{H} 07-\mathrm{Gal}$. myr-GFP marks $\mathrm{R} 92 \mathrm{H} 07-\mathrm{TuBu}$ neurons. Projections from a small population of nonTuBu neurons are also labelled at very low levels. mtdTomato (mtdTom) labels post-synaptic neurons. Note overlap of myr-GFP and mtdTomato signals in the superior bulb (arrow), with projections from downstream neurons innervating the ellipsoid body rings (EB, arrow) (I). EB rings innervated by R2-/R4d-/R5-neurons are shown (I).

Scale bars: $50 \mu \mathrm{m}$. See also Figure S3. 


\section{ACKNOWLEDGEMENTS}

We thank Gilad Barnea, Kyunghee Koh, Francois Rouyer and Ralf Stanewsky for Drosophila stocks; and Ko-Fan Chen, Simon Lowe, Kyunghee Koh and Francois Rouyer for helpful comments on the manuscript. Ko-Fan Chen provided technical input relating to live-imaging and driver lines labelling the AOTU. This study was supported by a UCL Start-Up Fund to J.E.C.J.

\section{AUTHOR CONTRIBUTIONS}

Conceptualization: A.L and J.E.C.J. Methodology: A.L. Software: P.K.

Validation: A.L. Formal Analysis: A.L and P.K. Investigation: A.L. Writing Original Draft: J.E.C.J and A.L. Writing - Review and Editing: A.L, P.K and J.E.C.J. Visualisation: A.L, P.K and J.E.C.J. Supervision: J.E.C.J. Project Administration: J.E.C.J. Funding Acquisition: J.E.C.J.

\section{DECLARATION OF INTERESTS}

The authors declare no competing interests. 


\section{STAR METHODS}

\section{CONTACT FOR REAGENT AND RESOURCE SHARING}

Further information and requests for resources and reagents should be directed to and will be fulfilled by the Lead Contact, James Jepson (j.jepson@ucl.ac.uk)

\section{EXPERIMENTAL MODEL AND SUBJECT DETAILS}

Fly strains and crosses were reared on standard yeast-containing fly flood at a constant temperature of $25^{\circ} \mathrm{C}$, housed under $12 \mathrm{~h}$ : $12 \mathrm{~h}$ light-dark cycles (LD). Drosophila lines used for behavioral analysis were outcrossed for a minimum of four generations into an isogenic (iso31) background. Individual 2-4 day old males were used in all behavioral experiments. per and per $^{\mathrm{S}}$ mutant lines were kind gifts from Francois Rouyer and Ralf Stanewsky. Trans-Tango lines were a kind gift from Gilad Barnea.

\section{METHOD DETAILS}

Behavioral assays

Individual males were loaded into glass tubes containing $2 \%$ agar and $4 \%$ sucrose. Sleep measurements were performed using the Drosophila Activity Monitor (DAM) system (Trikinetics, MA, USA) [6]. For all experiments shown in this manuscript, Trikinetics monitors were housed in temperatureand light-controlled incubators (LMS, UK) as described previously [3]. Sleep graphs derived from Trikinetics data were generated using GraphPad Prism 6. 
Immunohistochemistry

Adult male Drosophila brains were immuno-stained as described previously [27]. Brains were fixed in 4\% paraformaldehyde at RT for $20 \mathrm{~min}$, and blocked in 5\% goat serum at RT for $1 \mathrm{~h}$. Primary antibodies used were as follows: rabbit anti-DsRed (Clontech) - 1:2000; mouse anti-Bruchpilot (nc82, Developmental Studies Hybridoma Bank (DSHB)) - 1:200; chicken anti-GFP (ThermoFisher) - 1:1000. Alexa-fluor secondary antibodies (goat anti-rabbit 555, goat anti-chicken 488 and goat anti-mouse 647; ThermoFisher) were used at 1:2000 except for labelling anti-BRP where goat anti-mouse 647 at a dilution of 1:500 was used.

\section{Confocal imaging and optogenetics}

All confocal and optical imaging experiments were performed using an inverted Zeiss LSM 710 confocal microscope. For experiments involving CAMPARI, crosses were performed at $25^{\circ} \mathrm{C}$. Subsequently, 1-3 days old flies were entrained in LD $12-12$ cycles at $22^{\circ} \mathrm{C} .48 \mathrm{~h}$ prior to the experiment, adult male Drosophila were transferred to individual glass tubes to facilitate catching without $\mathrm{CO}_{2}$ or cold-induced anaesthesia. On the experimental day, individual flies were manually caught and brains were subsequently dissected in HL3.1 buffer, of composition: $70 \mathrm{mM} \mathrm{NaCl}, 5 \mathrm{mM}$ $\mathrm{KCl}, 20 \mathrm{mM} \mathrm{MgCl}$, $1.5 \mathrm{mM} \mathrm{CaCl}$, $10 \mathrm{mM} \mathrm{NaHCO}_{3}, 5 \mathrm{mM}$ Trehalose, 115mM sucrose, 5mM HEPES, pH 7.2. UV exposure was undertaken using a high power mercury lamp for 2 min. When testing for changes in CAMPARI photo-conversion following a rise in ambient temperature from $22^{\circ} \mathrm{C}$ to $31^{\circ} \mathrm{C}$, the dissection buffer was pre-heated to $31^{\circ} \mathrm{C}$ in order to 
maintain the experimental temperature. For experiments involving

GCamP6s, brains were dissected in the same dissection buffer as above.

To excite GCamP6s, a $514 \mathrm{~nm}$ laser was used, whereas to activate

ChR2XXL, a 405nm laser was applied.

\section{QUANTIFICATION AND STATISTICAL ANALYSIS}

Since many of the datasets derived from sleep experiments exhibited a non-normal distribution, the following statistical tests were used. For single comparisons, Mann-Whitney U-tests were used. When comparing multiple genotypes, Kruskal-Wallis tests were used, followed by Dunn's post-hoc tests. All statistical analyses were performed using GraphPad Prism 6. For each dataset, details of statistical tests used, n-values, dispersion and precision measures can be found in the corresponding Figure Legends.

\section{DATA AND SOFTWARE AVAILABILITY}

The custom-made R-based package for analysis of the duration, onset and offset of the longest sleep bout is detailed in, and can be downloaded from, the following Github link:

https://github.com/PatrickKratsch/DAM_sleep_parameters 


\section{REFERENCES}

1. Borbely, A.A., A two process model of sleep regulation. Hum Neurobiol, 1982. 1(3): p. 195-204.

2. Liu, S., et al., WIDE AWAKE mediates the circadian timing of sleep onset. Neuron, 2014. 82(1): p. 151-66.

3. Lamaze, A., et al., Regulation of sleep plasticity by a thermo-sensitive circuit in Drosophila. Sci Rep, 2017. 7: p. 40304.

4. Liu, S., et al., Sleep Drive Is Encoded by Neural Plastic Changes in a Dedicated Circuit. Cell, 2016. 165(6): p. 1347-1360.

5. Flourakis, M., et al., A Conserved Bicycle Model for Circadian Clock Control of Membrane Excitability. Cell, 2015. 162(4): p. 836-48.

6. Pfeiffenberger, C., et al., Processing sleep data created with the Drosophila Activity Monitoring (DAM) System. Cold Spring Harb Protoc, 2010. 2010(11): p. pdb prot5520.

7. Konopka, R.J. and S. Benzer, Clock mutants of Drosophila melanogaster. Proc Natl Acad Sci U S A, 1971. 68(9): p. 2112-6.

8. Kunst, M., et al., Calcitonin gene-related peptide neurons mediate sleep-specific circadian output in Drosophila. Curr Biol, 2014. 24(22): p. 2652-64.

9. Kitamoto, T., Conditional modification of behavior in Drosophila by targeted expression of a temperature-sensitive shibire allele in defined neurons. J Neurobiol, 2001. 47(2): p. 81-92.

10. Fosque, B.F., et al., Neural circuits. Labeling of active neural circuits in vivo with designed calcium integrators. Science, 2015. 347(6223): p. 755-60. 
11. Cavanaugh, D.J., et al., Identification of a circadian output circuit for rest:activity rhythms in Drosophila. Cell, 2014. 157(3): p. 689-701.

12. Zhang, L., et al., DN1(p) circadian neurons coordinate acute light and PDF inputs to produce robust daily behavior in Drosophila. Curr Biol, 2010. 20(7): p. 591-9.

13. Zhang, Y., et al., Light and temperature control the contribution of specific DN1 neurons to Drosophila circadian behavior. Curr Biol, 2010. 20(7): p. 600-5.

14. Omoto, J.J., et al., Visual Input to the Drosophila Central Complex by Developmentally and Functionally Distinct Neuronal Populations. Curr Biol, 2017. 27(8): p. 1098-1110.

15. Ilius, M., R. Wolf, and M. Heisenberg, The central complex of Drosophila melanogaster is involved in flight control: studies on mutants and mosaics of the gene ellipsoid body open. J Neurogenet, 1994. 9(3): p. 189-206.

16. Neuser, K., et al., Analysis of a spatial orientation memory in Drosophila. Nature, 2008. 453(7199): p. 1244-7.

17. Seelig, J.D. and V. Jayaraman, Feature detection and orientation tuning in the Drosophila central complex. Nature, 2013. 503(7475): p. 262-6.

18. Timaeus, L., L. Geid, and T. Hummel, A topographic visual pathway into the central brain of Drosophila. bioRxiv, 2018. https://www.biorxiv.org/content/early/2017/09/05/183707. 
19. Feinberg, E.H., et al., GFP Reconstitution Across Synaptic Partners (GRASP) defines cell contacts and synapses in living nervous systems. Neuron, 2008. 57(3): p. 353-63.

20. Chen, T.W., et al., Ultrasensitive fluorescent proteins for imaging neuronal activity. Nature, 2013. 499(7458): p. 295-300.

21. Dawydow, A., et al., Channelrhodopsin-2-XXL, a powerful optogenetic tool for low-light applications. Proc Natl Acad Sci U S A, 2014. 111(38): p. 13972-7.

22. Hamada, F.N., et al., An internal thermal sensor controlling temperature preference in Drosophila. Nature, 2008. 454(7201): p. 217-20.

23. Seidner, G., et al., Identification of Neurons with a Privileged Role in Sleep Homeostasis in Drosophila melanogaster. Curr Biol, 2015. 25(22): p. 2928-38.

24. Talay, M., et al., Transsynaptic Mapping of Second-Order Taste Neurons in Flies by trans-Tango. Neuron, 2017. 96(4): p. 783-795 e4.

25. Lebestky, T., et al., Two different forms of arousal in Drosophila are oppositely regulated by the dopamine $D 1$ receptor ortholog DopR via distinct neural circuits. Neuron, 2009. 64(4): p. 522-36.

26. Donlea, J.M., et al., Recurrent Circuitry for Balancing Sleep Need and Sleep. Neuron, 2018.

27. Wu, J.S. and L. Luo, A protocol for dissecting Drosophila melanogaster brains for live imaging or immunostaining. Nat Protoc, 2006. 1(4): p. 2110-5. 
Figure 1
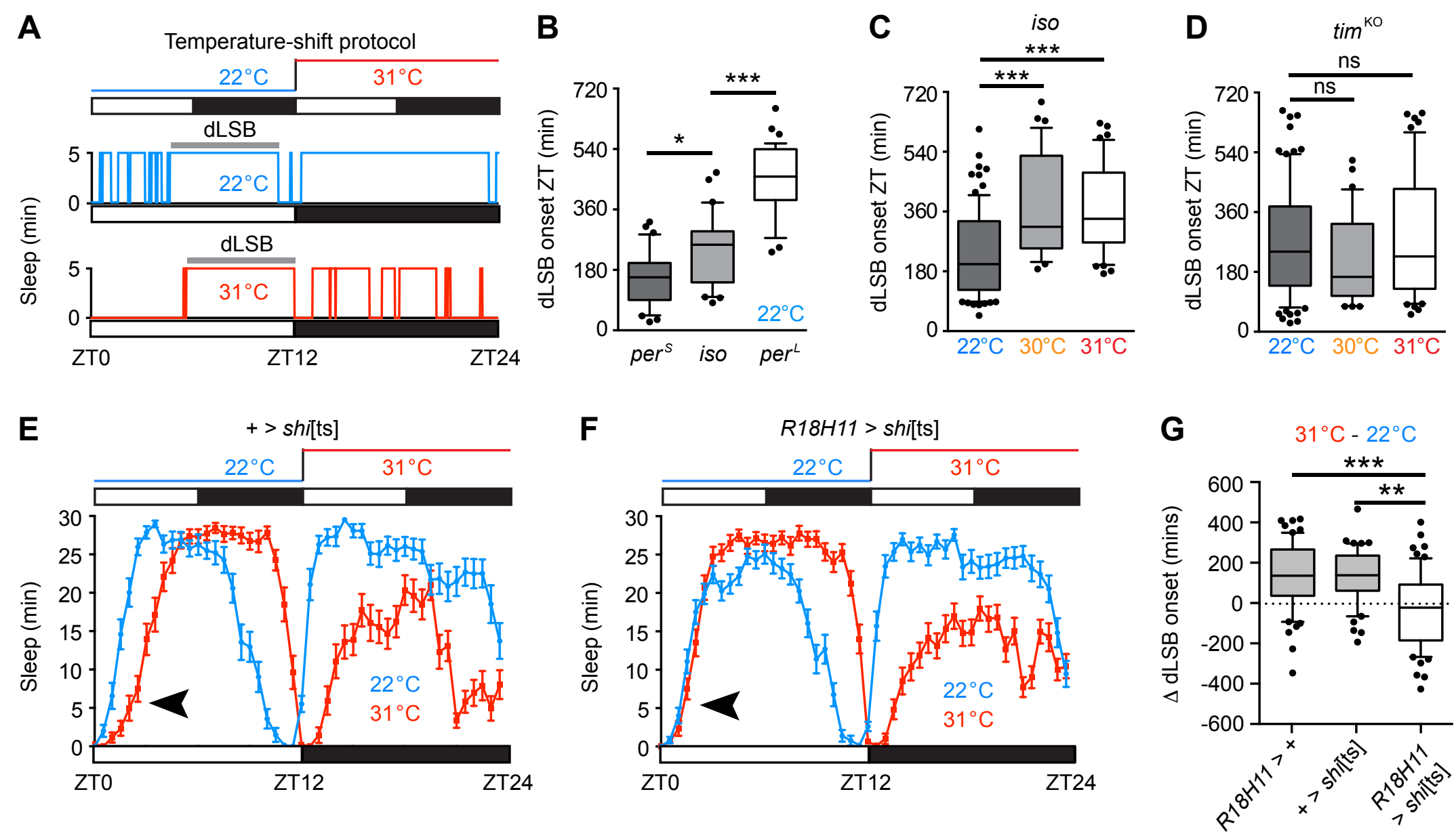

H
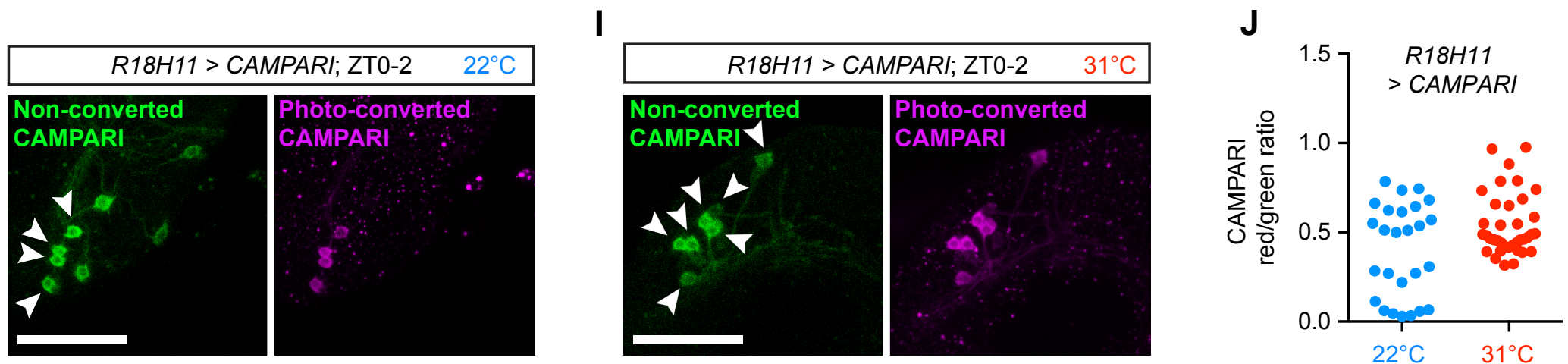
bioRxiv preprint doi: https://doi.org/10.1101/250829; this version posted January 22, 2018. The copyright holder for this preprint (which was not certified by peer review) is the author/funder, who has granted bioRxiv a license to display the preprint in perpetuity. It is made Figure 2 available under aCC-BY-NC-ND 4.0 International license.

A

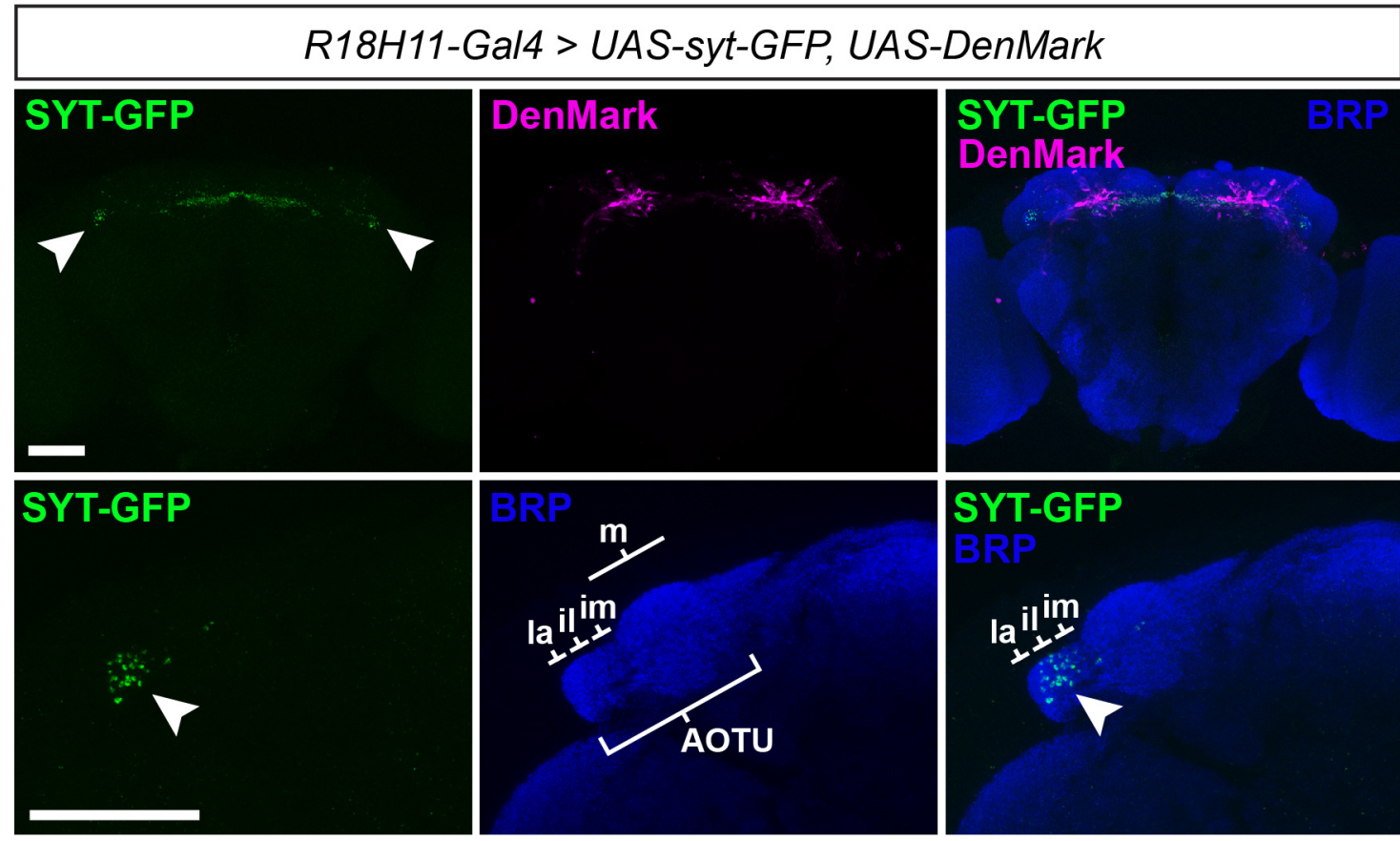

B

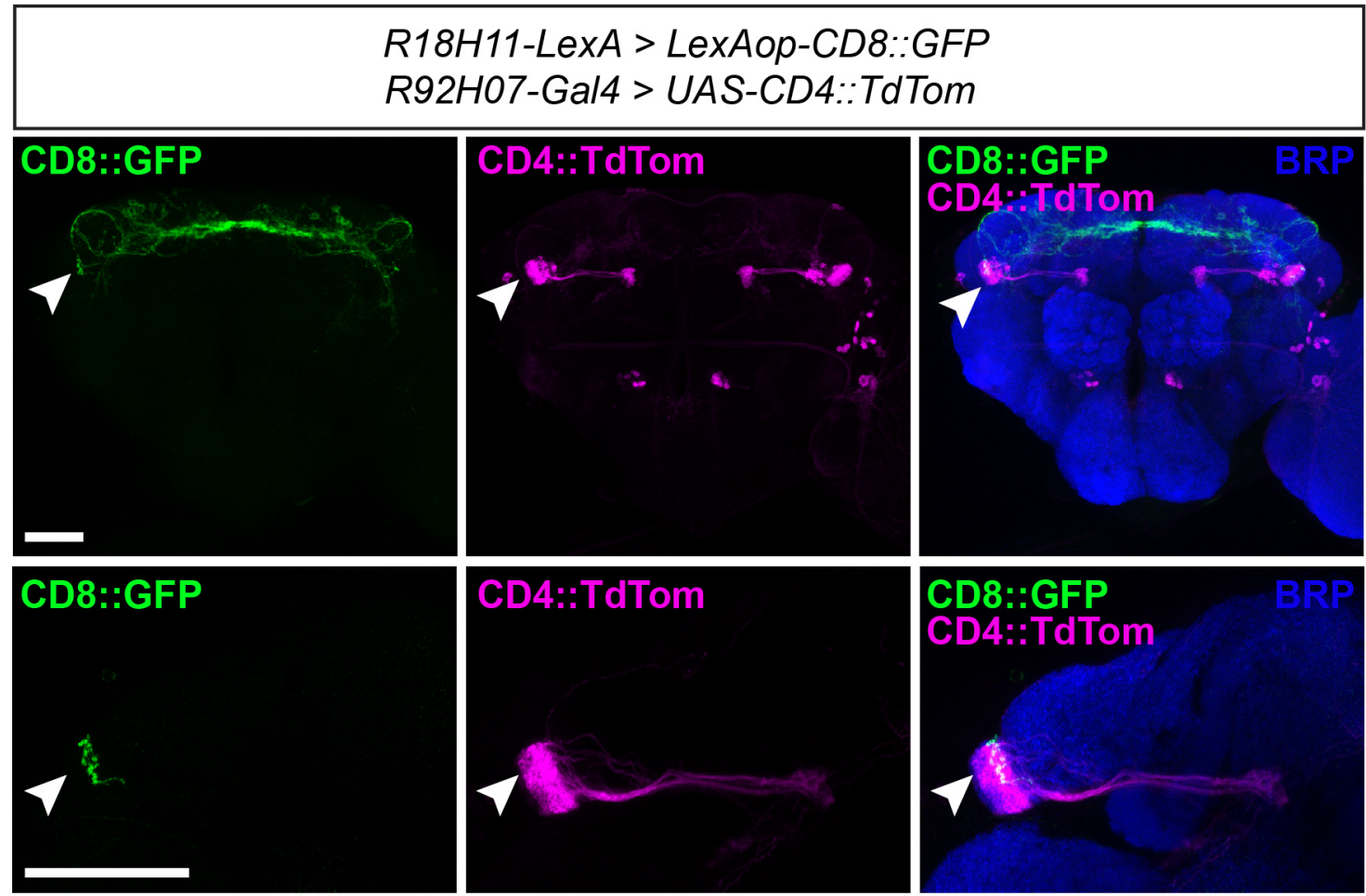

C

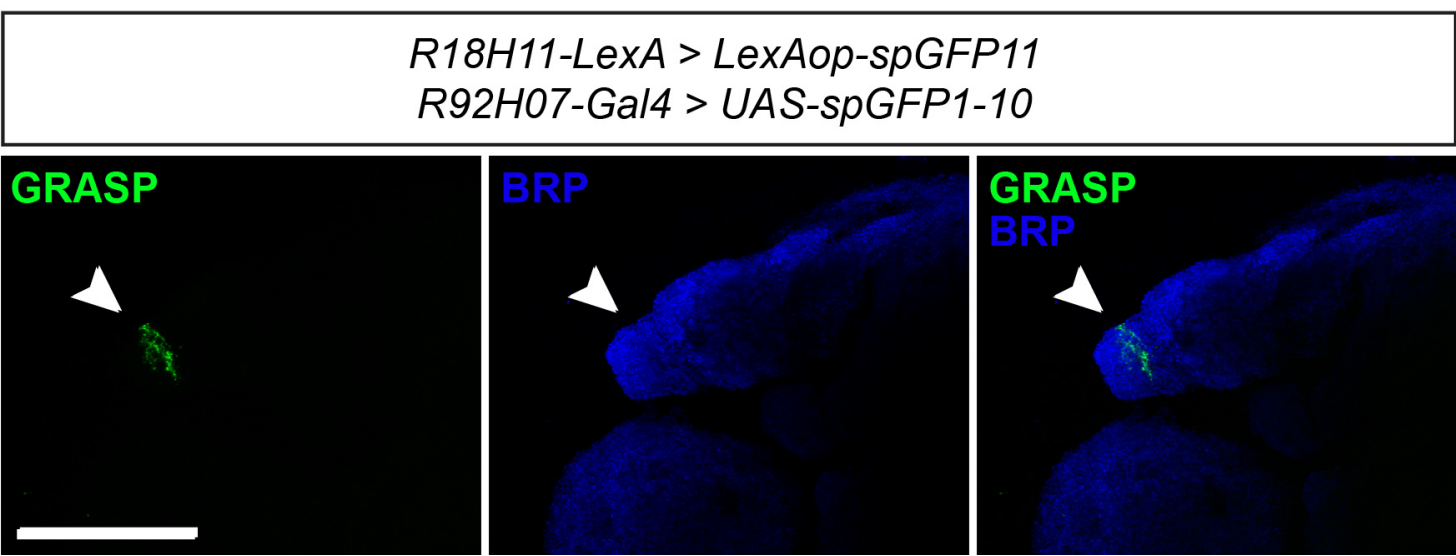




\section{Figure 3}

A $R 18 H 11>C A M P A R I$

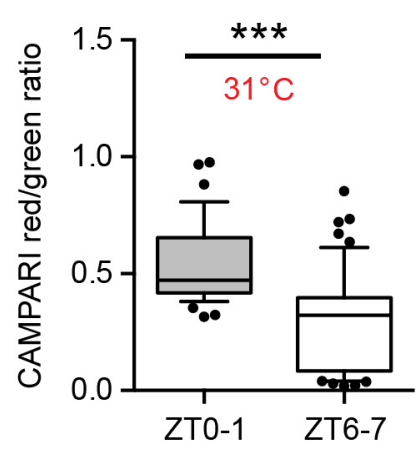

B $\quad$ R92HO7 > CAMPARI

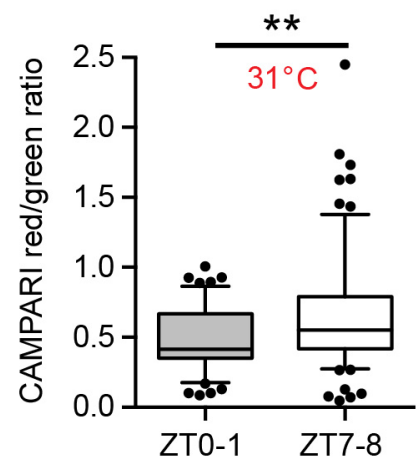

C

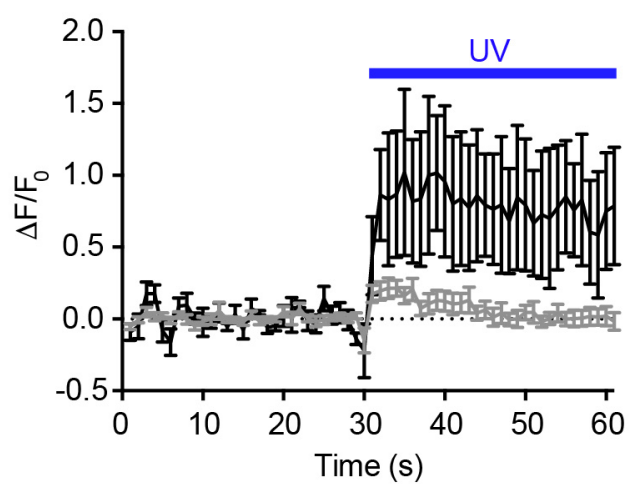

$R 18 H 11>+, \quad R 18 H 11>C h R 2-X X L$, R92H07 > GCamP6s R92H07> GCamP6s

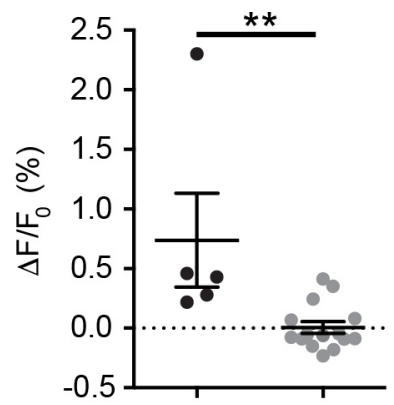

D

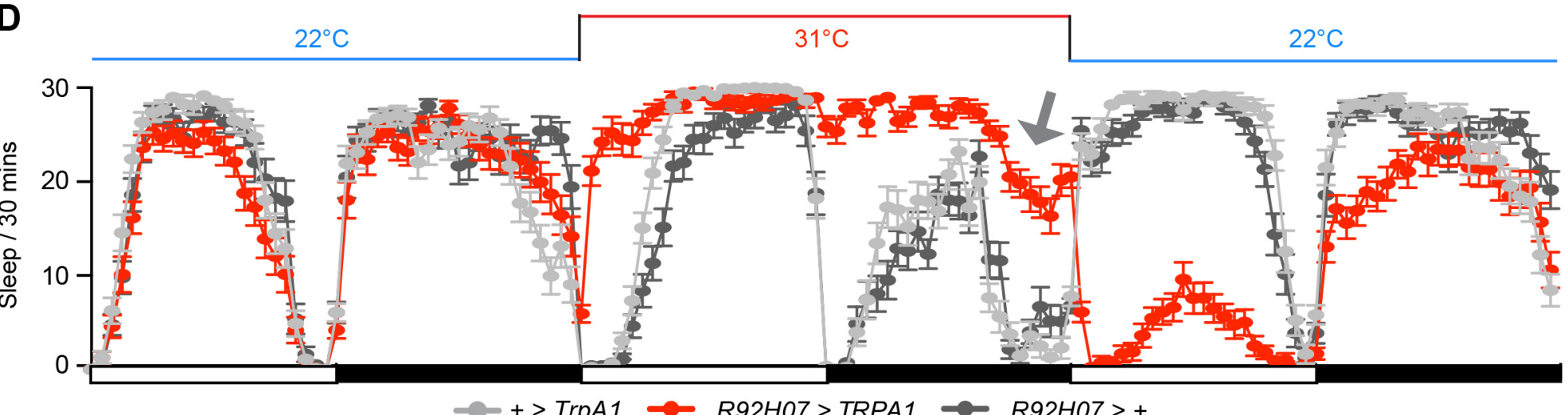

E

Day 2 - Day 1

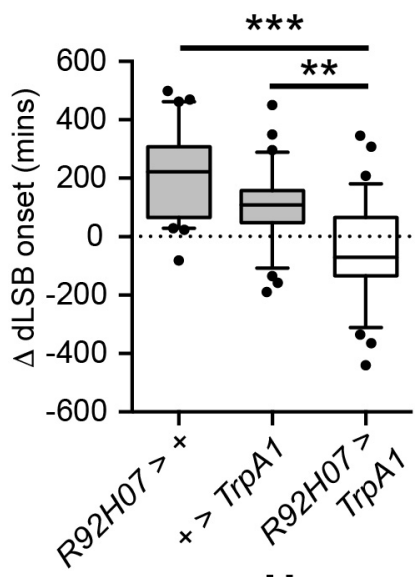

H
F Day 1 - Day 3

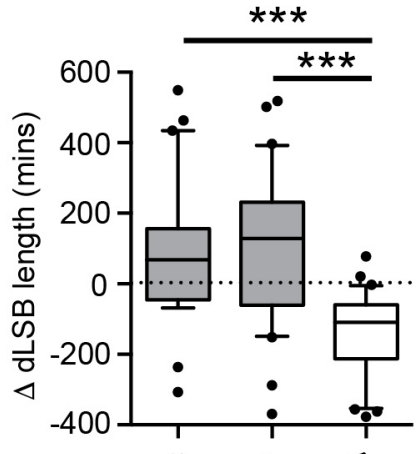

G

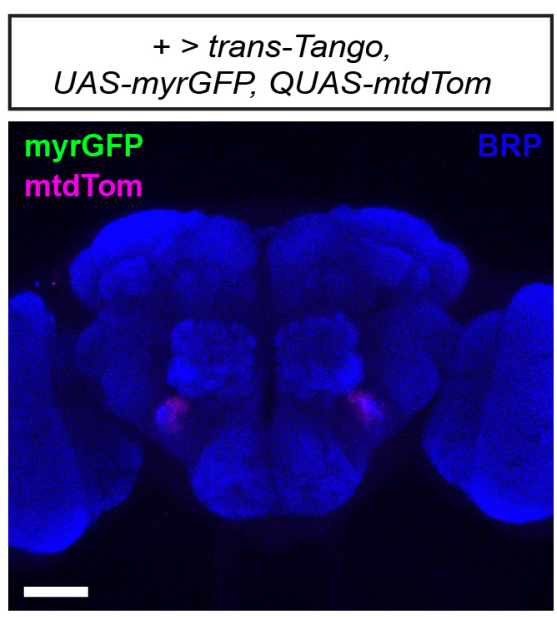

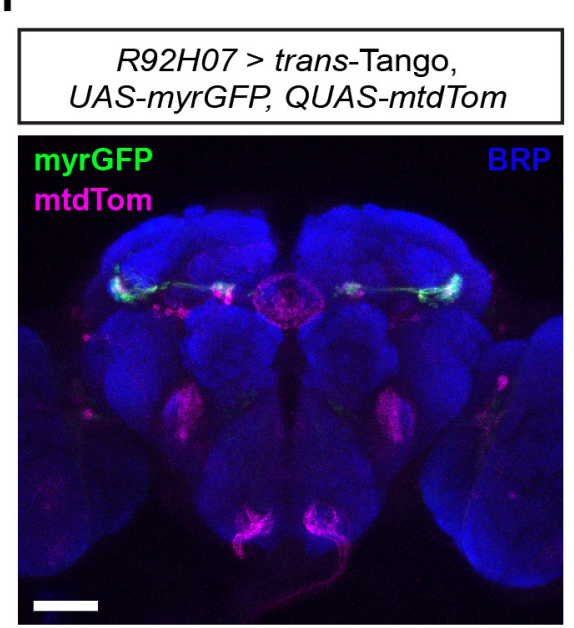

R92HO7 > trans-Tango, UAS-myrGFP, QUAS-mtdTom

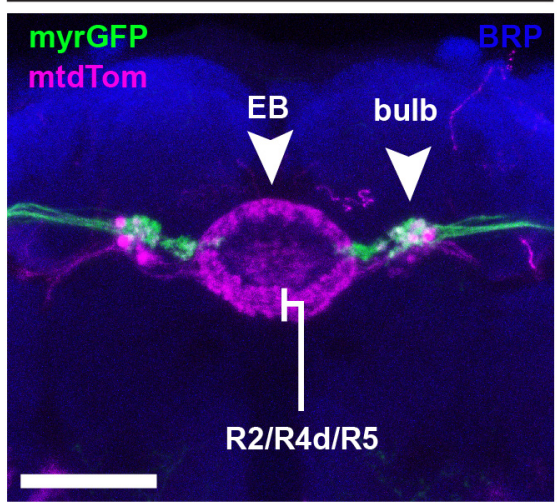




\section{Supplemental Information}

A sleep-regulatory circuit integrating circadian, homeostatic and environmental information in Drosophila

Angélique Lamaze, Patrick Krätschmer and James E. C. Jepson 
Figure S1

A
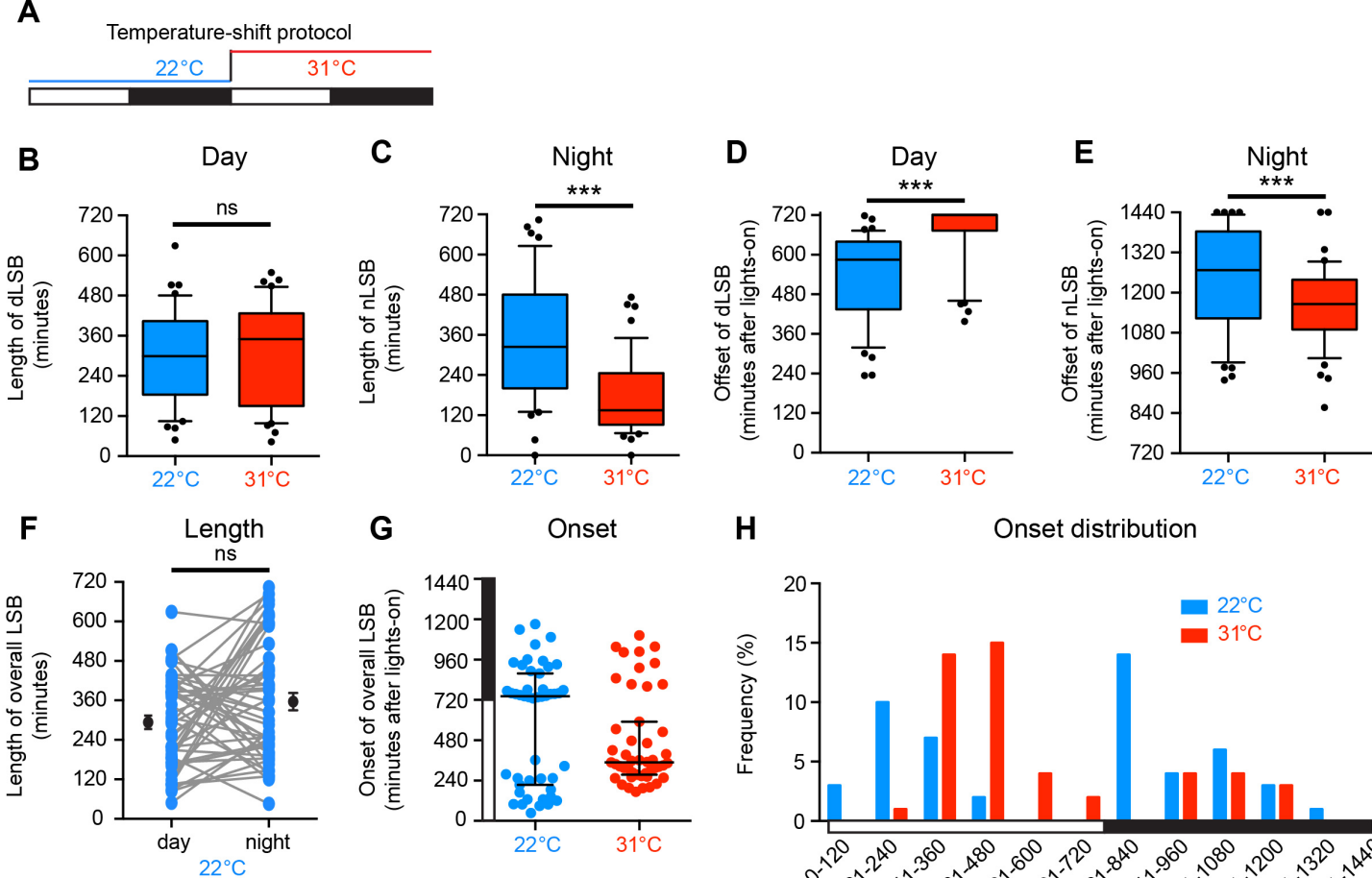

$\mathbf{H}$

Onset distribution
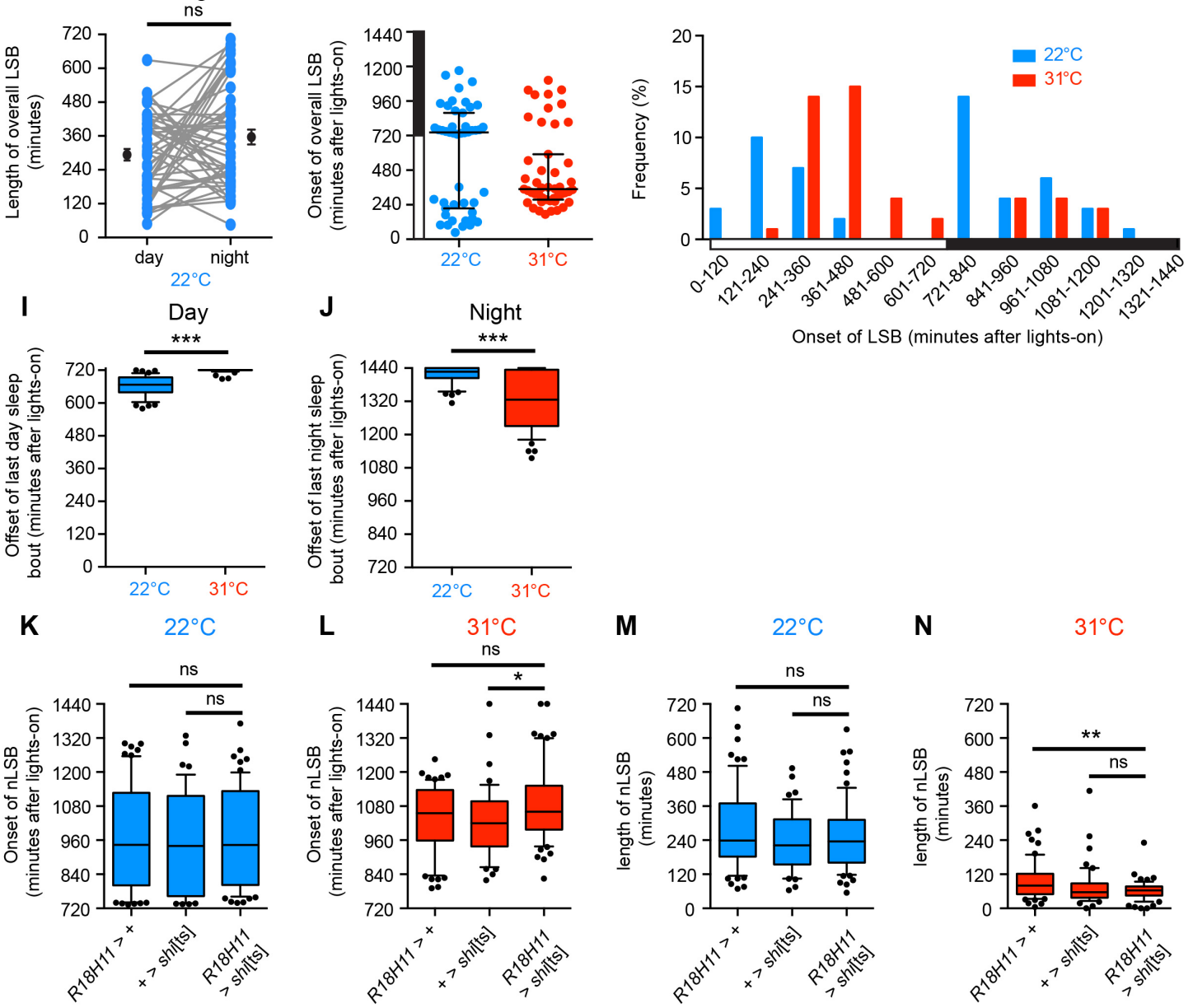

$\mathbf{N} \quad 31^{\circ} \mathrm{C}$

Figure S1, relating to Figure 1. Temperature-dependent changes in sleep architecture and role of DN1p clock neurons.

(A) Paradigm used to test how a range of parameters describing sleep architecture change in response to elevated temperature. On day 1 , adult male flies were housed at $22^{\circ} \mathrm{C}$, and on day 2 , ambient temperature was raised to $31^{\circ} \mathrm{C}$. White bars: day. Black bars: night. Activity was recorded on both days using the DAM system and the custom-made R-program was used 
to quantify sleep parameters described in (B-J). All data presented in (B-J) are derived from $n=47$ iso31 control adult male flies.

$(B-E)$ Changes in the length $(B-C)$ and time of offset $(D-E)$ of the longest sleep bouts (LSB) during the day (dLSB; $B, D$ ) or night ( $n L S B ; C, E$ ) in response to increased ambient temperature. Note that day and night sleep patterns respond differently to increased ambient temperature. The length of the daytime LSB is not changed (B), whereas the length of the night LSB is significantly reduced $(C)$. Furthermore, the offsets of the day and night LSBs are also altered in an opposing manner, with offset of the day LSB being delayed (D), and offset of the night LSB advanced (E), consistent with a reduced length of night LSB. The distribution of data is illustrated using Tukey box plots, as described in Figure 1.

(F) Paired comparison of the length of the day and night LSB in individual flies at $22^{\circ} \mathrm{C}$. Connecting bars indicate how these two parameters vary in individual flies. No significant difference in the average LSB between day and night was observed (black circle denotes mean \pm SEM for day and night LSBs). This is consistent with Drosophila exhibiting a crepuscular pattern of rest/activity. $(\mathrm{G}, \mathrm{H})$ Distributions showing the onset of the LSB across the complete $24 \mathrm{~h}$ period (overall LSB), shown via dots plots $(\mathrm{G})$ or histogram $(H)$. White bars: day. Black bars: night. Median and interquartile ranges are shown. As described above in $(F)$, at $22^{\circ} \mathrm{C}$ the day and night LSBs are not significantly different in length. Correspondingly, for a population of flies housed at $22^{\circ} \mathrm{C}$, the overall LSB during $24 \mathrm{~h}$ can be initiated during either the day or the night, but is most commonly observed directly after lights-off. However, at $31^{\circ} \mathrm{C}$ we observed that the overall LSB is more frequently initiated during the day, suggesting that at high temperatures flies shift from a crepuscular to a nocturnal pattern of activity.

$(\mathrm{I}, \mathrm{J})$ Offset of the last day $(\mathrm{I})$ and night $(\mathrm{J})$ sleep bout at either $22^{\circ} \mathrm{C}$ or $31^{\circ} \mathrm{C}$. Again, day and night sleep offset responds in an opposite manner to increased temperature, with the offset of the last day sleep bout being delayed, and the offset of the last night sleep bout advanced, at high ambient temperature.

$(\mathrm{K}-\mathrm{N})$ Comparison of the onset $(\mathrm{K}, \mathrm{L})$ and length $(\mathrm{M}, \mathrm{N})$ of the night longest sleep bout (nLSB) at either $22^{\circ} \mathrm{C}$ or $31^{\circ} \mathrm{C}$ in adult male flies expressing the temperature-sensitive inhibitor of synaptic vesicle endocytosis $(R 18 H 11>$ shi[ts]) and controls. $22^{\circ} \mathrm{C}(\mathrm{K}, \mathrm{M})$ : control condition in which dominantnegative temperature-sensitive shibire (shi[ts]) is inactive. $31^{\circ} \mathrm{C}(\mathrm{L}, \mathrm{N})$ : DN1p inhibition by shi[ts] $(R 18 H 11>$ shi[ts]) and control flies examined under the same temperature conditions. $R 18 H 11>+: \mathrm{n}=63 ;+>$ shi[ts]: $\mathrm{n}=50$; $R 18 H 11>$ shi[ts]: $\mathrm{n}=63$.

${ }^{*} p<0.05,{ }^{* *} p<0.01,{ }^{* * *} p<0.001$, ns $-p>0.05$, Mann-Whitney U-test (B-F, IJ) or Kruskal-Wallis test with Dunn's post-hoc test (K-N). 
bioRxiv preprint doi: https://doi.org/10.1101/250829; this version posted January 22, 2018. The copyright holder for this preprint (which was not certified by peer review) is the author/funder, who has granted bioRxiv a license to display the preprint in perpetuity. It is made available under aCC-BY-NC-ND 4.0 International license.

\section{Figure S2}

A

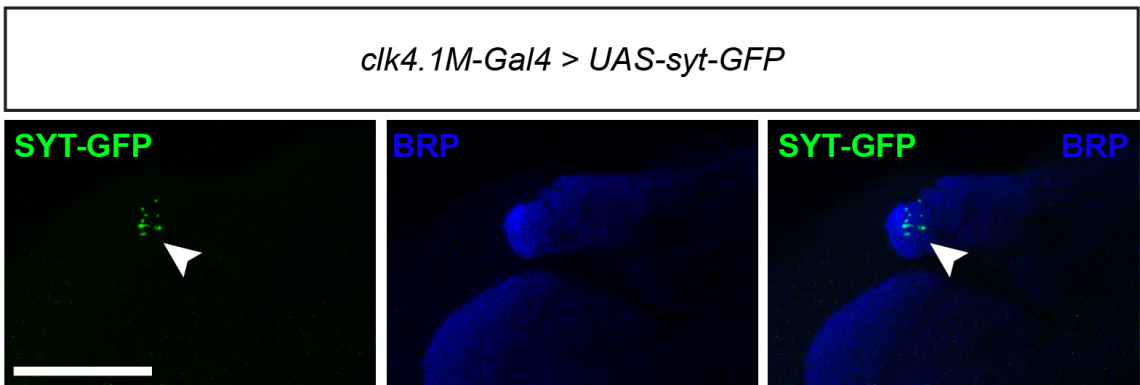

B

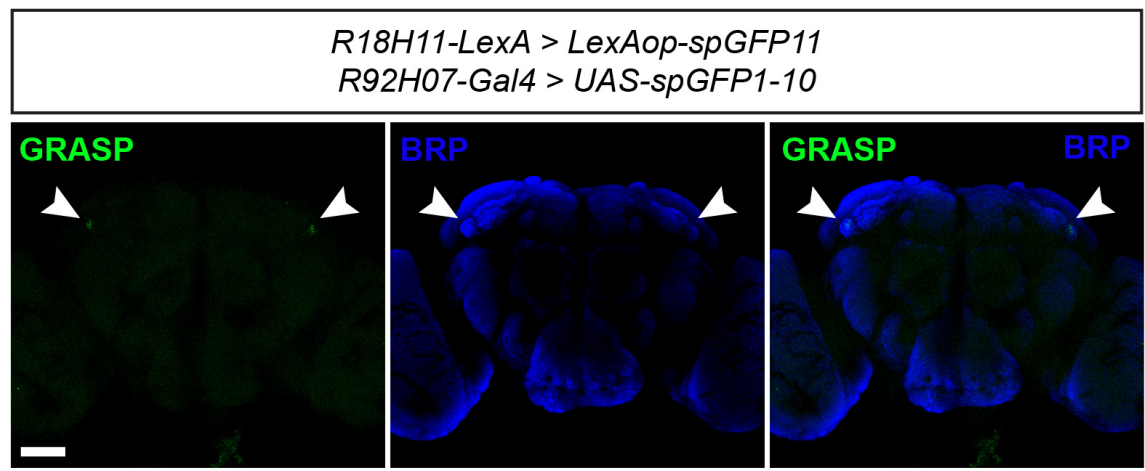

C
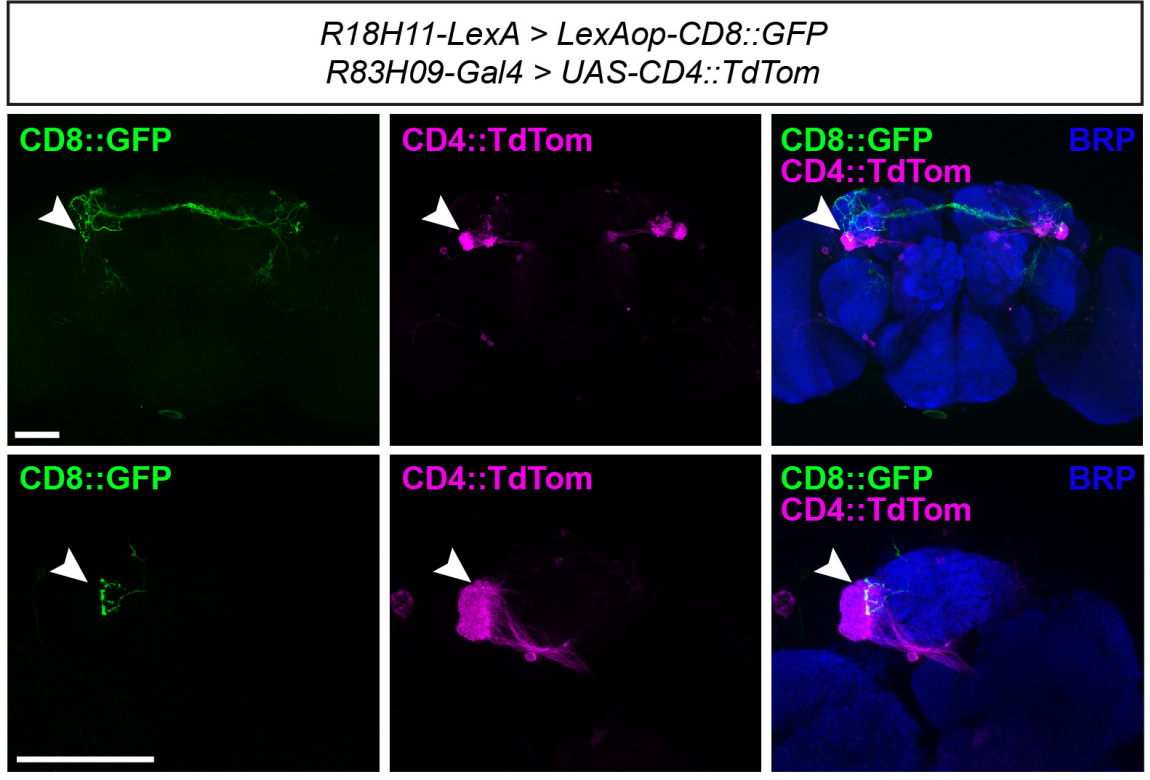

D

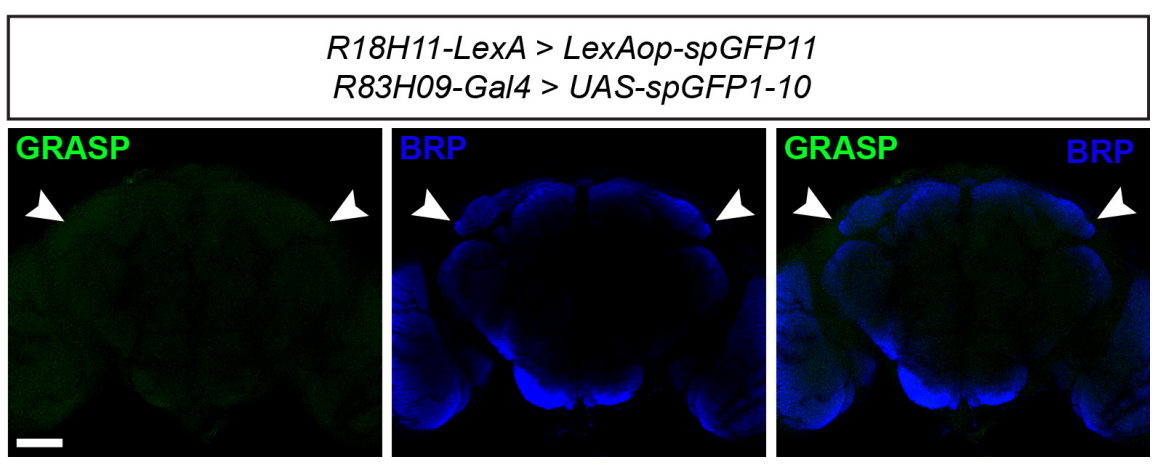


Figure S2, relating to Figure 2. DN1p neurons make synaptic connections with TuBu neurons in the AOTU.

(A) Confocal images showing localisation of presynaptic SYT-GFP puncta derived from clk4.1M-Gal4-expressing adult male DN1p neurons. Arrows point to SYT-GFP puncta within the anterior optic tubercle (AOTU). BRP: Bruchpilot (a presynaptic neuropil marker).

(B) Confocal images showing that GRASP signal obtained through expression of split-GFP fragments in DN1p neurons (via R18H11-LexA) and TuBu neurons (via R92H07-Gal4) is detected specifically in the AOTU (arrows), and not elsewhere in the central brain.

(C) Dual labelling of DN1p and R83H09-positive TuBu neurons that innervate the lateral AOTU. Arrows point to DN1p presynaptic termini that tile the boundary of the lateral AOTU, and thus the dendritic domain of R83H09-TuBu neurons.

(D) Confocal images showing lack of GRASP signal from split-GFP fragments expressed in DN1p neurons (via R18H11-LexA) and R83H09-TuBu neurons. Arrows point to the AOTU region. No GRASP signal was detected here or elsewhere in the Drosophila brain (compare with Figure S2A).

All scale bars: $50 \mu \mathrm{m}$. 


\section{Figure S3}

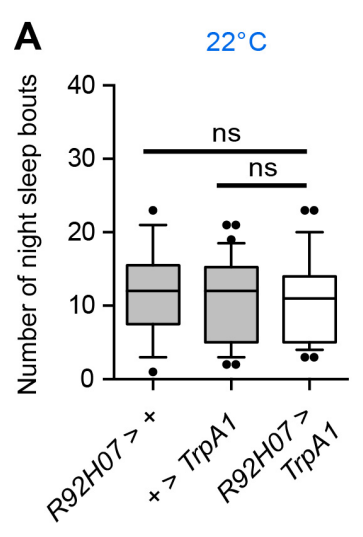

D

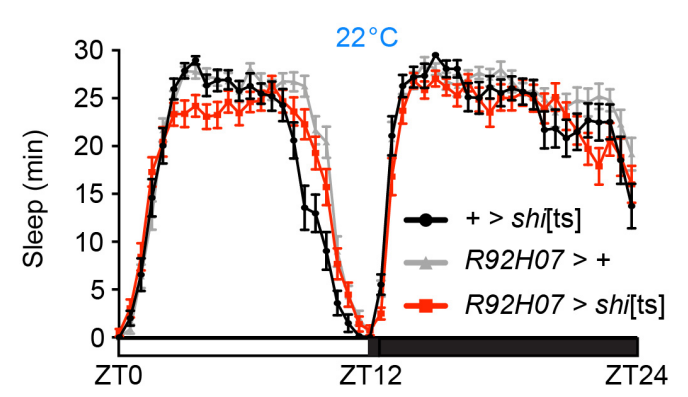

$\mathbf{G}$

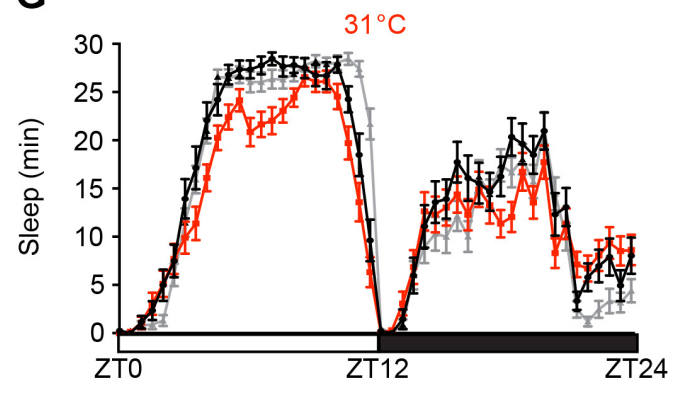

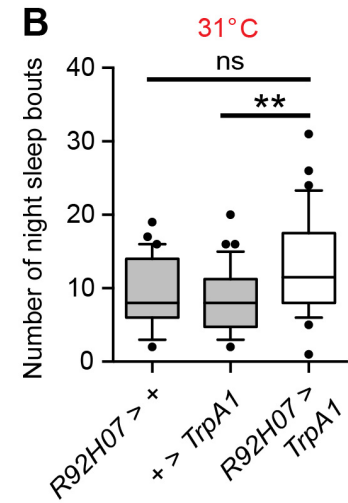

C

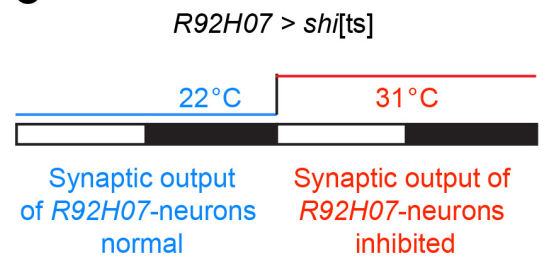

E

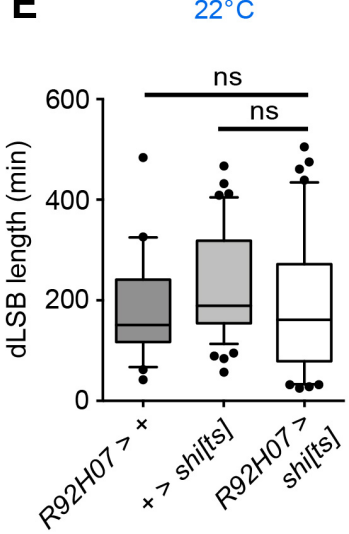

$\mathbf{F}$
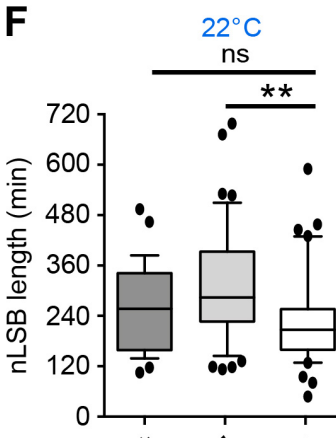

H

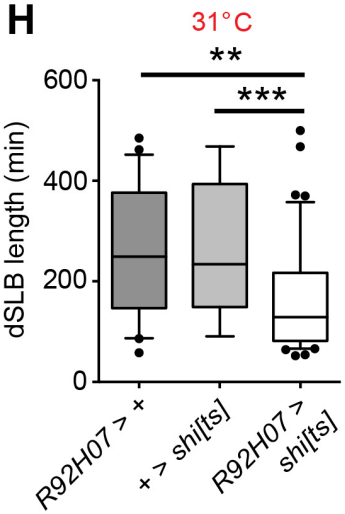

I

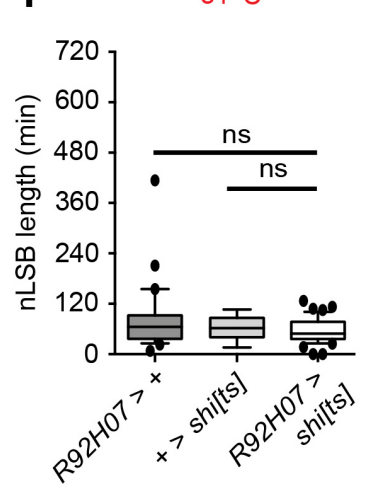

Figure S3, relating to Figure 3. Activation of $\mathrm{R} 92 \mathrm{H07-TuBu}$ neurons does not cause paralysis.

(A-B) Thermogenetic activation of R92H07-TuBu neurons does not alter the number of sleep bouts during the night. Sleep levels in flies expressing the TrpA1 thermo-sensory cation channel were measured on two consecutive days. On day $1(\mathrm{~A})$, ambient temperature was $22^{\circ} \mathrm{C}$, a non-activating temperature for $\operatorname{TrpA} 1$. On day $2(\mathrm{~B})$, ambient temperature was $31^{\circ} \mathrm{C}$, an activating temperature. Relative to both control lines, no change in sleep bout number during the night was apparent. Thus, when R92H07-TuBu neurons are activated, flies are still able to awaken and initiate movement, demonstrating that they are not paralysed. $R 92 H 07>+: \mathrm{n}=29 ;+>\operatorname{TrpA} 1: \mathrm{n}$ $=34 ; R 92 H 07>\operatorname{TrpA} 1: \mathrm{n}=36$. 
(C) Paradigm for acute inhibition of $\mathrm{R} 92 \mathrm{H} 07-\mathrm{TuBu}$ neurons at elevated temperatures using shi[ts].

$(\mathrm{D}-\mathrm{F})$ At the permissive temperature of $22^{\circ} \mathrm{C}$, the length of the longest day (dSLB) and night (nSLB) sleep bouts were unaffected by expression of shi[ts].

(G-I) At $31^{\circ} \mathrm{C}$, inhibition of synaptic release from $\mathrm{R} 92 \mathrm{H} 07-\mathrm{TuBu}$ neurons results in a significant reduction in length of the dLSB but not the $\mathrm{nLSB}$.

R92H07 > +: $\mathrm{n}=45 ;+>$ shi[ts]: $\mathrm{n}=29 ;$ R92H07 > shi[ts]: $\mathrm{n}=41$.

${ }^{* *} p<0.01,{ }^{* * *} p<0.001$, ns $-p>0.05$, Kruskal-Wallis test with Dunn's posthoc test. 